\title{
STATIONARY POINT FREE GROUP ACTIONS
}

\author{
R. E. STONG
}

1. Introduction. The object of this paper is to establish the result:

THEOREM. If $\alpha \in \Omega_{*}$ is a torsion element of the oriented cobordism ring $\Omega_{*}$, then there is an oriented manifold $M$ representing the cobordism class $\alpha$ together with an action $\phi$ of $S^{1}$ on $M$ by orientation preserving diffeomorphisms having the property that $\phi(z, m)=m, z \in S^{1}, m \in M$, implies $z$ is either 1 or -1 .

Denote by $S F(G), G$ a compact Lie group, the ideal of classes $\alpha \in \Omega_{*}$ represented by an oriented manifold $M$ on which $G$ acts by orientation preserving diffeomorphisms with the action having no stationary points. One then has:

Corollary. If $T^{k}$ is the toroidal group $S^{1} \times \cdots \times S^{1}$ (k-times), then $S F\left(T^{k}\right)$ is the torsion ideal of $\Omega_{*}$.

This result was conjectured by Conner and Floyd [2, p. 131] and was proved by P. G. Anderson (Thesis, Massachusetts Institute of Technology, Cambridge). Conner and Floyd proved in Corollary (43.8) that $S F\left(T^{k}\right) \subset$ Torsion $\left(\Omega_{*}\right)$. Denoting by $\pi: T^{k} \rightarrow S^{1}$ the projection on the first factor and $\phi: S^{1} \times M \rightarrow M$ the action given by the theorem,

$$
T^{k} \times M \stackrel{\pi \times 1}{\longrightarrow} S^{1} \times M \stackrel{\phi}{\longrightarrow} M
$$

is a stationary point free action of $T^{k}$ on $M$. Thus Torsion $\left(\Omega_{*}\right)$ $C S F\left(T^{k}\right)$.

One also has the result conjectured by Conner and Floyd [2, p. 143]:

Corollary. For $k \geqq 2$, the ideal $S F\left(Z_{2}{ }^{k}\right)$ is precisely the ideal of classes $\alpha \in \Omega_{*}$ having even Euler characteristic.

The author is indebted to the National Science Foundation for financial support during this work and to Oxford University for their hospitality during part of this work.

2. Proof of the theorem. In order to prove the theorem, note first that if $M$ is an oriented manifold and $\phi: S^{1} \times M \rightarrow M$ is an $S^{1}$ action, then for each $t \in S^{1}, \phi(t, \quad): M \rightarrow M$ is an orientation preserving diffeomorphism since it is homotopic to the identity. Thus the orientation preserving property is automatic for $S^{1}$ actions.

Received by the editors September 23, 1966. 
Next, recall from Wall [4] that the algebra $W_{*}$ of unoriented cobordism classes represented by a manifold with $w_{1}$ reduced integral is a polynomial algebra over $Z_{2}$ on classes $x_{2 k-1}, x_{2 k}, x_{2} j^{2}$ with $k$ not a power of 2 . There is a homomorphism $\partial: \varpi_{n+1} \rightarrow \Omega_{n}$ obtained by sending the class of $M$ into the class of the submanifold $N$ of $M$ dual to $w_{1}$, and Wall has shown that image $(\partial)$ is precisely the set of torsion elements of $\Omega_{*}$.

Using Wall's representative manifolds, one has that the classes $x_{2 k-1}$ and $x_{2^{j}}^{2}$ contain orientable manifolds, and since $\partial$ is a derivation, for any monomial

$$
Y=\prod_{\alpha} x_{2}^{2 j} \cdot \prod_{\beta} x_{2 k_{\beta}-1} \cdot \prod_{\gamma} x_{2 k_{\gamma}}
$$

that

$$
\partial Y=\prod_{\alpha} x_{2}^{2}{ }^{j \alpha} \cdot \prod_{\beta} x_{2 k_{\beta}-1} \cdot \partial\left\{\prod_{\gamma} x_{2 k_{\gamma}}\right\} .
$$

Thus, it suffices to show that each class $\partial\left\{x_{2 k_{1}} \cdots x_{2 k_{r}}\right\}$ contains a representative manifold on which $S^{1}$ acts in the desired fashion. There are two cases to be considered: $r=1$ and $r>1$.

First, consider the case $r=1$. Then $\partial x_{2 k}=x_{2 k-1}$ is representable by a Dold manifold $P(m, n)$ with $m$ odd and $n$ even (see [3]). In particular, $P(m, n)$ is obtained from $S^{m} \times C P(n)$ by identifying the points $(x, \eta)$ and $(-x, \bar{\eta})$. Letting $m=2 p-1$, define

$$
\begin{aligned}
S^{1} \times S^{m} \times C P(n) & \rightarrow S^{m} \times C P(n):\left(z,\left(z_{1}, \cdots, z_{p}\right), \eta\right) \\
& \rightarrow\left(\left(z z_{1}, \cdots, z z_{p}\right), \eta\right)
\end{aligned}
$$

where $S^{1} \subset C^{1}, S^{m} \subset C^{p}$ in the usual way. This preserves identifications to give

$$
\phi: S^{1} \times P(m, n) \rightarrow P(m, n)
$$

and $\phi(z, u)=u$ implies $z$ is either 1 or -1 . This gives an action of $S^{1}$ on $P(m, n)$ of the desired type.

For the case $r>1$, one wants a representative for $\partial\left(x_{2 k_{1}} \cdots x_{2 k_{r}}\right)$. For this recall that a representative for $x_{2 k}$ is obtained as follows: $P(m, n)$ has an involution

$$
\tau: P(m, n) \rightarrow P(m, n)
$$

induced by the involution

$$
\begin{aligned}
S^{m} \times C P(n) & \rightarrow S^{m} \times C P(n):\left(\left(z_{1}, \cdots, z_{p}\right), \eta\right) \\
& \rightarrow\left(\left(-z_{1}, \cdots,-z_{p-1},-\bar{z}_{p}\right), \eta\right)
\end{aligned}
$$


given by the reflection of $S^{m}$ in the plane for which the last coordinate is zero. Then let $Q(m, n)$ be formed from $S^{1} \times P(m, n)$ by identifying $(t, u)$ and $(-t, \tau(u))$. As noted by Wall, $Q(m, n)$ represents $x_{2 k}$ (for properly chosen $m$ and $n$ ).

Let $N_{i}=Q\left(m_{i}, n_{i}\right)$ represent $x_{2 k_{i}}, i=1, \cdots, r$. Let $\pi_{i}: N_{i} \rightarrow S^{1}$ be the projection induced by $S^{1} \times P\left(m_{i}, n_{i}\right) \rightarrow S^{1}:(t, u) \rightarrow t^{2}$. Then $\pi_{i}$ is a fibration and realizes $w_{1}\left(N_{i}\right)$. Let $S^{1}$ act on $N_{i}$ by means of the action

$$
S^{1} \times S^{1} \times P\left(m_{i}, n_{i}\right) \rightarrow S^{1} \times P\left(m_{i}, n_{i}\right):(z, t, u) \rightarrow(z t, u) .
$$

The action $\phi_{i}: S^{1} \times N_{i} \rightarrow N_{i}$ has fixed points only for 1 and -1 in $S^{1}$.

Now let $P: N_{1} \times \cdots \times N_{r} \rightarrow S^{1}:\left(v_{1}, \cdots, v_{r}\right) \rightarrow\left(\pi_{1}\left(v_{1}\right) \cdots \pi_{r}\left(v_{r}\right)\right)$. This is the composition of the bundle maps

$$
\pi_{1} \times \cdots \times \pi_{r}: N_{1} \times \cdots \times N_{r} \rightarrow S^{1} \times \cdots \times S^{1}
$$

and

$$
\mu: S^{1} \times \cdots \times S^{1} \rightarrow S^{1}
$$

where $\mu$ is the multiplication. Thus $P$ is transverse regular on $1 \in S^{1}$. In addition, $P$ realizes $w_{1}$, so

$$
V=\left\{\left(v_{1}, \cdots, v_{r}\right) \in N_{1} \times \cdots \times N_{r} \mid \pi_{1}\left(v_{1}\right) \cdots \pi_{r}\left(v_{r}\right)=1\right\}
$$

represents $\partial\left(x_{2 k_{1}} \cdots x_{2 k_{r}}\right)$. Note. This step was suggested by the proof of Anderson [1].

Let $\phi: S^{1} \times V \rightarrow V:\left(z,\left(v_{1}, \cdots, v_{r}\right)\right) \rightarrow\left(\phi_{1}\left(z^{-1}, v_{1}\right), v_{2}, \cdots, v_{r-1}\right.$, $\left.\phi_{r}\left(z, v_{r}\right)\right)$. Then $\phi$ is an action of $S^{1}$ and $\phi(z, v)=v$ implies $z$ is 1 or -1 . Thus $\partial\left(x_{2 k_{1}} \cdots x_{2 k_{r}}\right)$ is represented by $V$ on which $S^{1}$ acts in the desired manner.

\section{Actions of $Z_{2}$.}

Proposition. Let $Z_{2^{k}}, k \geqq 1$, act on $M$ without stationary points Then the Euler characteristic, $\chi(M)$, is even.

Proof. One inducts on $k$. Let $F \subset M$ be the set of points fixed under the action of $Z_{2} \subset Z_{2}$. By [2, (27.2)], $\chi(M) \equiv \chi(F) \bmod 2$. Then the action of $Z_{2}$ on $F$ factors through an action of $Z_{2^{k-1}}$, so $Z_{2}{ }^{k-1}$ acts on $F$ without stationary points.

To prove the corollary, it then suffices to show that

$$
S F\left(Z_{4}\right) \supset\left\{\alpha \in \Omega_{*} \mid \chi(\alpha) \equiv 0(\bmod 2)\right\} .
$$

First note that $Z_{2}$ acts freely on $2 M$ by interchanging components, so $S F\left(Z_{4}\right) \supset S F\left(Z_{2}\right) \supset 2 \Omega_{*}$ and by the theorem $S F\left(Z_{4}\right) \supset$ Torsion $\left(\Omega_{*}\right)$. Since $\Omega_{*} /\left(2 \Omega_{*}+\right.$ Torsion $\left.\Omega_{*}\right)$ is isomorphic to the $Z_{2}$ polynomial algebra on the classes of the complex projective spaces $C P(2 k)$, it suffices to 
show that some manifold cobordant to $C P(2 k) \pm C P(2)^{k}$ has a $Z_{4}$ action by orientation preserving diffeomorphisms with no stationary points.

For this, one may follow Conner and Floyd [2, p. 142]. One has a $Z_{4}$ action on $C P(2 k)$ given by

$$
T\left(\left[z_{0}, z_{1}, z_{2}, \cdots, z_{2 k-1}, z_{2 k}\right]\right)=\left[\bar{z}_{0},-\bar{z}_{2}, \bar{z}_{1}, \cdots,-\bar{z}_{2 k}, \bar{z}_{2 k-1}\right]
$$

with $T^{4}=1 . T$ is orientation preserving since any diffeomorphism of $C P(2 k)$ preserves orientation. Further, the only stationary point of $T$ is $[1,0, \cdots, 0]$. Then $T \times \cdots \times T$ acts on $C P(2)^{k}$ with one stationary point, and the action at this point is the same as the action at the stationary point of $T$ on $C P(2 k)$. Deleting neighborhoods of these points and gluing together along the resulting boundaries gives a manifold $V^{4 k}$ cobordant to $C P(2 k) \pm C P(2)^{k}$ on which $Z_{4}$ acts as required.

\section{REFERENCES}

1. P. G. Anderson, Cobordism classes of squares of orientable manifolds, Ann. of Math. 83 (1966), 47-53.

2. P. E. Conner and E. E. Floyd, Differentiable periodic maps, Springer-Verlag, Berlin, 1964.

3. A. Dold, Vollständigkeit der Wu-schen Relationen zwischen den Stiefel-Whitneyschen Zahlen differenzierbarer Mannigfaltigkeiten, Math. Z. 65 (1956), 200-206.

4. C. T. C. Wall, Determination of the cobordism ring, Ann. of Math. 72 (1960), 292-311.

Princeton University 